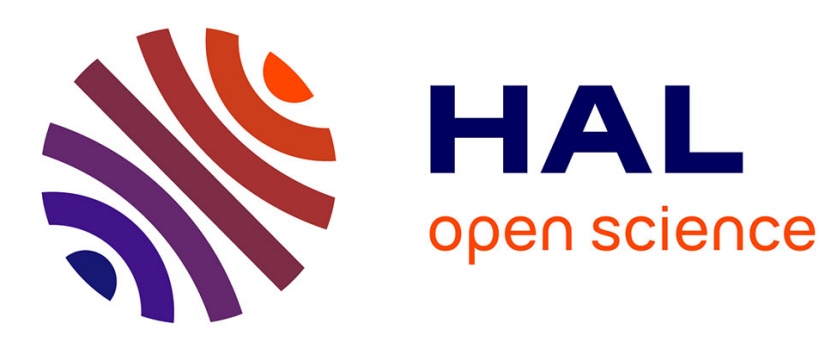

\title{
CRITIC: A Cognitive Radio Inspired Road Traffic Congestion Reduction Solution
}

Soufiene Djahel, Adam Jones, Yassine Hadjadj-Aoul, Ashfaq Khokhar

\section{To cite this version:}

Soufiene Djahel, Adam Jones, Yassine Hadjadj-Aoul, Ashfaq Khokhar. CRITIC: A Cognitive Radio Inspired Road Traffic Congestion Reduction Solution. WD 2018 - 10th Wireless Days Conference, Apr 2018, Dubai, United Arab Emirates. pp.151-157, 10.1109/WD.2018.8361711 • hal-01934058

\section{HAL Id: hal-01934058 \\ https://hal.inria.fr/hal-01934058}

Submitted on 25 Nov 2018

HAL is a multi-disciplinary open access archive for the deposit and dissemination of scientific research documents, whether they are published or not. The documents may come from teaching and research institutions in France or abroad, or from public or private research centers.
L'archive ouverte pluridisciplinaire HAL, est destinée au dépôt et à la diffusion de documents scientifiques de niveau recherche, publiés ou non, émanant des établissements d'enseignement et de recherche français ou étrangers, des laboratoires publics ou privés. 


\title{
CRITIC: A Cognitive Radio Inspired Road Traffic Congestion Reduction Solution
}

\author{
Soufiene Djahel ${ }^{\mp}$, Adam Jones ${ }^{\mp}$, Yassine Hadjadj-Aoul ${ }^{\ddagger}$ and Ashfaq Khokhar $^{ \pm}$ \\ ${ }^{\mp}$ School of Computing, Mathematics and Digital Technologies, Manchester Metropolitan University, UK \\ ${ }^{\ddagger}$ IRISA/University of Rennes 1, France \\ ${ }^{ \pm}$Department of Electrical and Computer Engineering, Iowa State University, USA
}

\begin{abstract}
According to recent forecasts, constant population growth and urbanization will bring an additional load of 2.9 billion vehicles to road networks by 2050 . This will certainly lead to increased air pollution concerns, highly congested roads putting more strain on an already deteriorated infrastructure, and may increase the risk of accidents on the roads as well. Therefore, to face these issues we need not only to promote the usage of smarter and greener means of transportation but also to design advanced solutions that leverage the capabilities of these means along with modern cities' road infrastructure to maximize its utility. To this end, we propose an original Cognitive Radio inspired algorithm, named CRITIC, that aims to mimic the principle of Cognitive Radio technology used in wireless networks on road networks. The key idea behind CRITIC is to temporarily grant regular vehicles access to priority (e.g., bus or carpool) lanes whenever they are underutilized in order to reduce road traffic congestion. The performance evaluation results of CRITIC using grid and random road network topologies with varying traffic load have proven its effectiveness.
\end{abstract}

Keywords - Smart Transportation, Smart Cities, Road Traffic Congestion, Cognitive Radio, SUMO, TraCI.

\section{INTRODUCTION}

Traffic congestion is a significant problem that many cities have to face in the modern world. Indeed, the accelerated economic growth in urban areas has led to an unprecedented increase in population, creating plethora of challenges to efficiently accommodate their needs of fast mobility while ensuring their safety and minimizing the negative impact of transport means on the environment. From transport managers perspective, this generally equates to a larger number of private vehicles using the limited network of roads [1]. Many cities do not have the capacity, logistics or funding resources to cope with this increase in demand, which results in a degradation of the road infrastructure and ultimately increased traffic congestion problems on road networks.

Despite the numerous studies that have highlighted the negative impact of traffic congestion on the economy, environment and city inhabitants' safety and health, and the endless number of attempts to design and deploy sophisticated systems to reduce this impact, traffic congestion is still a serious issue that is both hard to accurately predict and efficiently overcome because it is influenced by several factors. The Victoria Transport Policy Institute, which is an independent think tank of traffic congestion research, defined some metrics that can be used to measure traffic-congestion, such as Level-Of-Service (LOS) and Travel-Time-Index (TTI). However, these metrics are only useful for measuring the current traffic congestion level and not for predicting its occurrence in a future context [2].

Due to the nature of traffic congestion, it has been shown to have a direct effect on the average travel times of all users on a road network, which naturally leads to delayed or canceled events, meetings and scheduled deliveries. A study conducted by INRIX, a leading traffic investigation service, and backed by the Texas Transportation Institute's 2012 Urban Mobility Report [3], has shown that between the years 2013 and 2030 the expected economic loss due to traffic congestion in the UK alone is estimated to be a staggering $£ 307$ billion, further reporting that this roughly equates to an increased congestion per-household cost from $£ 1,426$ in 2013 to $£ 2,057$ in 2030 .

Such factors further lead to an increase in stress level of individual drivers, which has been strongly correlated to drivers' aggression [4], which implies an increased probability of road traffic incidents due to lapse in concentration or poor judgments. In [5], the authors investigated the effects of stress and aggression caused by traffic congestion on the health of city inhabitants and attempted to measure the effects of stress on the health of individual drivers using an impedance measurement [6]. This study highlighted that an increased impedance measurement leads to higher stress levels, which could contribute to poor mental and physical health. Alongside its impact on individuals' physical health, traffic congestion also contributes to the poor natural environmental conditions, such as poor air and water conditions due to increased pollution. This is mainly because a tremendous percentage of private vehicles still using fossil fuels as their primary power source. When a fossil fuel is burnt, it releases toxic biproducts into the environment, which once coupled with traffic congestion leads to an excessive production of these toxins, worsening the quality of life in dense urban areas.

As discussed above, traffic congestion is a colossal problem in particular for big cities, but it also has wider implications in every aspect of modern society. Although there has been many research attempts for designing effective solutions to mitigate this problem and its negative impact, we are still a 
long way from meeting the reality of a truly 'smart city' where road networks can dynamically cope with the ever increasing demand on the road infrastructure. Therefore, to shorten this way, we propose an original solution that consists in applying the principle of wireless networks based technology into road networks to ultimately reduce the commuters' travel time and improve their travel experience. This technology is known as 'Cognitive Radio' introduced in [13] and its key advantage consists in offering opportunistic access to additional bandwidth for wireless devices so that their achieved throughput can be increased. Likewise, we propose to apply the principle of this technology in road networks so that vehicles can opportunistically gain access to reserved lanes (e.g. bus and carpool lanes) so that their travel time is reduced.

The remainder of this paper is organized as follows. In Section II, we provide a detailed description of our proposed solution. Section III presents and analyzes the performance evaluation results. Finally, Section IV concludes the paper.

\section{Proposed Solution}

In this section, we propose our original Cognitive Radio Inspired TraffIc Congestion reduction solution dubbed CRITIC in order to better manage traffic on road networks. CRITIC is based on a centralized approach in which a central traffic management entity such as a Traffic Light Controller (TLC) [8] monitors the traffic conditions (i.e. the occupancy level) on each incoming or outgoing lane that it controls, then CRITIC algorithm is triggered whenever its requirements are met. These requirements can be summarized into the following two conditions: (i) a reserved (or priority) lane, such as bus lanes or High-Occupancy Vehicle lane (aka carpool or diamond lane), is deemed to be underutilized, and (ii) traffic on other regular lanes is slowed down due to recurrent or non-recurrent congestion. In addition to monitoring traffic conditions, the TLC communicates with the vehicles as well and decide about the most effective lane guidance strategy for each of them to follow. The ultimate goal is to maximize the usage efficiency of the current road infrastructure without violating any local regulations.

\section{A. Understanding Cognitive Radio Principle}

In order to implement an effective algorithm that inherits from the ideas behind Cognitive Radio (CR) we must first understand how $\mathrm{CR}$ functions, and how its basic principals can be applied to the traffic management domain. In [9], the authors discussed the basic principles of a CR system by focusing on the following three key points that characterize such a system.

- Acquiring knowledge of its operational and geographical environment, established policies, and its internal state (i.e. cognitive capability).

- Based on the above knowledge, dynamic and autonomous adjustment of its operational parameters and protocols is performed to fulfill a set of predefined objectives (i.e. reconfigurable capability).

- Learn from the achieved results (i.e. learning capability).

The first point, discusses how a CR system attempts to analyze the current network state to ensure it can understand the key information needed to monitor the network performance and discover what features it can manipulate to improve it. An example of this would be to firstly calculate how busy the current network frequency band is, in order to gauge whether or not it is becoming congested. This is directly applicable to a road network scenario, the same concept could be applied to the lanes of each road, finding out which lanes are congested and taking the appropriate action to reduce the traffic congestion.

The second point highlights that once the network state is analyzed and necessary information are acquired, a CR system proceeds with optimizing/adapting some network parameters to improve the overall performance and reduce the congestion state. In wireless networks context, this could be the adaptation to another radio frequency band that is currently underutilized, in order to more efficiently utilize the whole radio spectrum, and ultimately improving the achieved throughput. Again, the same types of optimization can be applied to a road network, a traffic analyzer (i.e. monitor) could, first, estimate the congestion level in each lane on a road segment, and then try utilizing other lanes on this road segment more efficiently, for example allowing non-priority vehicles to use a priority lane if this latter is under-utilized.

The final point mentioned attempts to provide a way for CR system to be able to learn from the information it has gathered from the two previous steps, in order to better service the network in future circumstances. An example of this in road networks would be to keep track of times when priority vehicles were affected by regular vehicles using priority lanes, trying to better understand what variables caused this to happen. In future circumstances the CR algorithm can then try to avoid these scenarios, making the algorithm more efficient and causing less delay to priority vehicles.

\section{B. Cognitive Radio like Road Network Management}

In our solution, we aim to implement the first two key points presented by $\mathrm{CR}$ systems specification, which are acquiring knowledge of the geographical environment and dynamically adjusting its operational parameters. Implementing the final stage of a CR system is, however, out of scope of this paper and will be investigated in a future work.

Figure 1 depicts the different actions performed by the TLC as part of CRITIC system. The TLC must continuously monitor the road network to retrieve and analyze the current traffic conditions, enabling the detection of any under-utilized priority lane or a congested non-priority lane. CRITIC is then triggered to mimic a CR system once an under-utilized priority lane and a congested regular lane are detected. In order to mimic a CR 


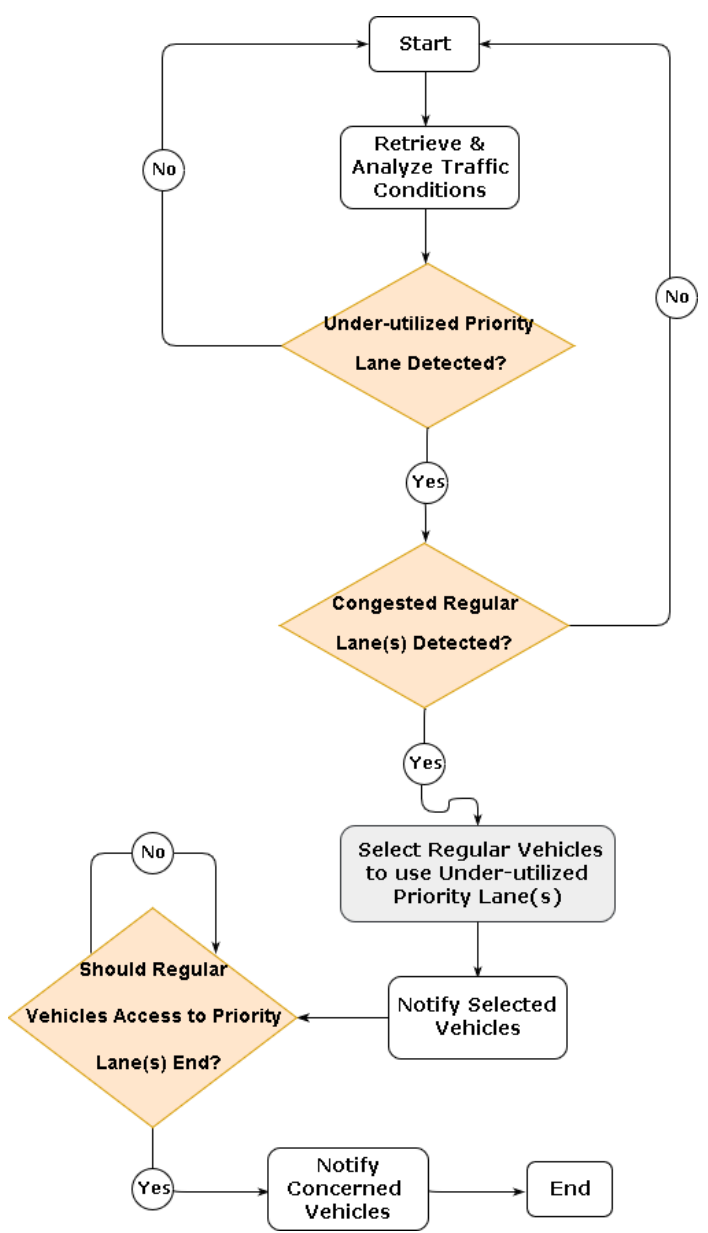

Figure 1: A flow chart modeling TLC actions within CRITIC

system, CRITIC algorithm should select a subset of regular vehicles, as discussed later in Section II-C, and notify them that they now have permission to use the priority lane, either for a fixed period of time or until further notice.

Figure 2 describes the actions taken by a non-priority vehicle upon receiving the selection notification from the TLC. The foremost feature a user needs to ensure is that no collision occurs during the time taken to move to a new lane, this is an additional feature that is required compared with the traditional $\mathrm{CR}$ algorithm, as in terms of virtual networks, collisions are not as harmful as in a real physical road network. Once a vehicle receives a notification from the TLC it must first ensure that it is safe to change its lane by coordinating its actions with the neighboring vehicles in both lanes (i.e. its current lane and the priority lane that this vehicle will move to). To safely achieve this a coordination protocol like [10] or [11] can be used. Afterward, the vehicle can use the priority lane till the expiration of its temporary access period or upon revocation of this access permission, in which case a safe lane change can be made back to a non-priority lane.

Figure 3 shows a more in depth illustration of the similarities between a CR system and CRITIC, on the left hand side of

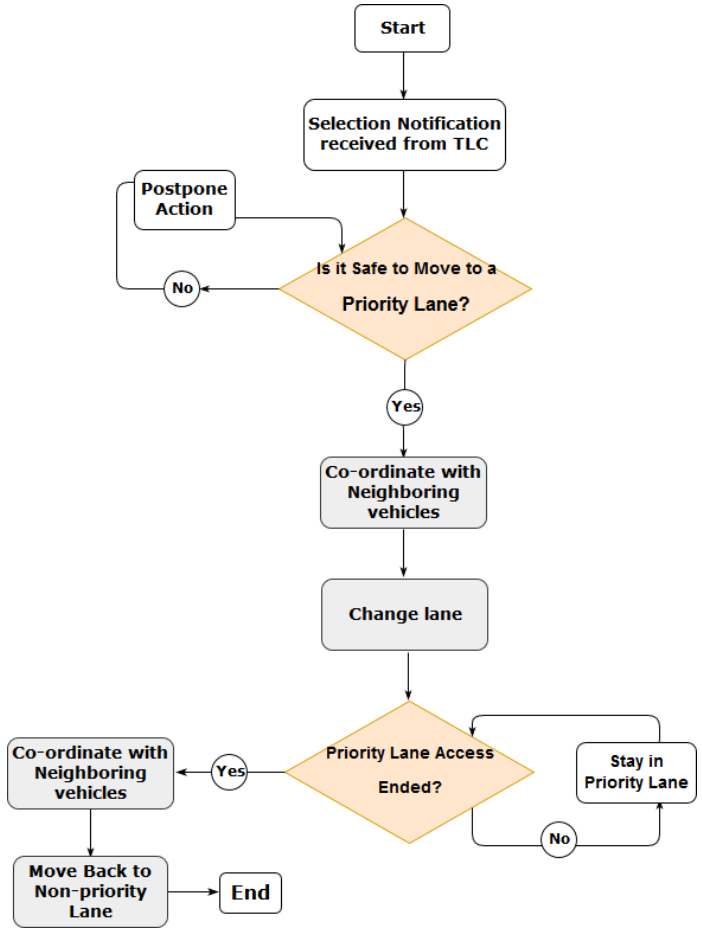

Figure 2: A flow chart modeling a vehicle actions within CRITIC

the diagram the picture depicts what a CR network would look like and the actions taken by a CR system to optimize the usage of the available frequency bands, by allowing users of the congested spectrum 2 to utilize the detected spectrum holes in the frequency band 1 (as secondary users). The right hand side of this figure shows how well this concept can be applied to a road network with priority lanes, here traffic lane 2 is highly congested and the CRITIC algorithm allows a number of selected vehicles to temporarily utilize the underutilized bus lane to alleviate the current congestion in other lanes.

\section{Vehicles Selection Criteria}

Once the above requirements are met, the TLC selects a subset of regular vehicles in the congested lanes to move to the under-utilized priority lane(s) for either a fixed period of time or till further notice. This step is very crucial as it has a significant impact on the efficiency of CRITIC. First, we need to determine how many vehicles should we select (i.e. Subset $t_{\text {size }}$ ), then which vehicles to be selected, and for how long they will be allowed to use the priority lane. The number of vehicles to be selected depends on the length of the priority lane(s) and congestion level of non-priority lane(s), a simple way to determine this number is shown in Eq. 3 . In this equation two inputs are used; (i) Capacity (see Eq. 1) defining the number of vehicles that the priority lane can host, and (ii) Reduction (see Eq. 2) which represents the number of additional vehicles that created the congestion. 


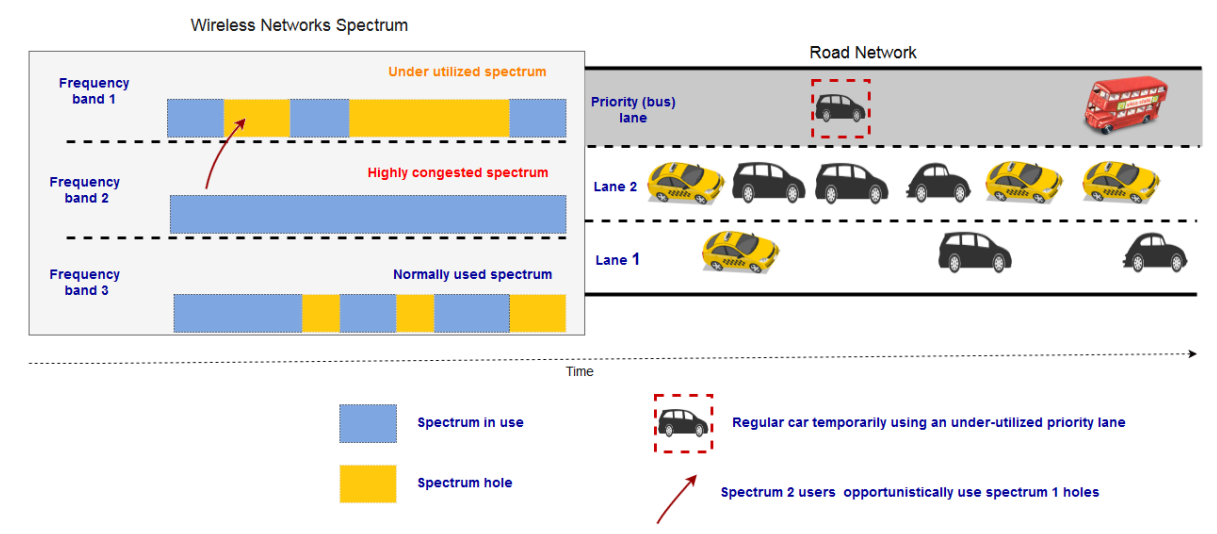

Figure 3: Illustration of CR functionality on the roads

$$
\begin{gathered}
\text { Capacity }=\frac{\text { Length }(P L)+\text { Safety Distance }}{A V G_{V L}+\text { Safety Distance }} \\
\text { Reduction }=\left(\frac{F F S_{R L}-A V G_{\text {Speed }}(R L)}{F F S_{R L}}\right) \times \# \text { Vehicles }
\end{gathered}
$$

$$
\text { Subset }_{\text {size }}=\min (\text { Capacity, Reduction })
$$

Where Length $(P L)$ refers to the length of the priority lane, $A V G_{V L}$ is the average length of a vehicle, $F F S_{R L}$ represents the Free Flow Speed of the regular congested lane while $A V G_{\text {Speed }}(R L)$ denotes its current average speed. It is worth to mention as well that the Safety Distance is computed based on the maximum speed allowed in the priority lane.

The selection of vehicles that will be temporarily permitted to use a priority lane is made first based on their power source (i.e. electricity, hybrid and fossil fuel) and then their trip length, meaning that electric vehicles with longer trips will have the highest probability (i.e., $\operatorname{Pr}(i)$ value for a vehicle $i$ ) to use the priority lane, as shown in Equations 4 and 5.

$\operatorname{Pr}(i)=\left(W_{\text {power }} \times \operatorname{Power}_{\text {value }}(i)\right)+\left(W_{\text {trip }} \times \operatorname{Trip}_{\text {value }}(i)\right)$

such that Power $_{\text {value }}$ is set to $1,0.8$ and 0.7 for electric, hybrid and fossil fuel vehicles, respectively. $\operatorname{Trip}_{\text {value }}(i)$ is a normalized value representing the remaining distance of the vehicle $i$ trip and calculated according to Eq. 5. Note that $W_{\text {power }}$ and $W_{\text {trip }}$ are weight values both equal to 0.5 .

$$
\operatorname{Trip}_{\text {value }}(i)=\frac{\operatorname{Trip}_{i}}{\text { max }_{\text {trip }}}
$$

where Trip $_{i}$ denotes the remaining distance to the destination for the vehicle $i$, while $\max _{\text {trip }}$ represents the maximum value of this distance among all the vehicles using the congested regular lane.

Now, the TLC selects the vehicles with the highest $\operatorname{Pr}(i)$ values (i.e. the top subset $_{\text {size }}$ vehicles) and notify them to change their current lane(s) and move to the under-utilized priority lane(s). Notice that for safety and efficiency purposes, the TLC may choose to notify a lower number of vehicles than the selected subset $_{\text {size }}$ vehicles, mainly based on their current positions within each lane.

\section{Performance evaluation}

In this section we present the performance evaluation results of CRITIC algorithm under two different representative road layouts: Grid and Abstract road networks. The simulation was performed using SUMO ${ }^{1}$ and TraCi in which we implemented the code of CRITIC using Python.

\section{A. Evaluation metrics}

The metrics used to evaluate CRITIC's performance are Average Travel Time (ATT) and Travel Time Index (TTI). The ATT is the mean travel time of all vehicle trips, it is a useful metric for determining the overall status of the road network [7]. The travel time (TT) is the time spent on the road network by a vehicle to reach its destination. ATT is computed as shown in Eq. 6, where $n$ is the total number of vehicles and $T T$ is the travel time.

$$
A T T=\frac{\sum_{i=1}^{n} T T_{i}}{n}
$$

TTI is the ratio of the TT during peak hours compared to the free flow TT [12]. Free flow TT is the time needed for a vehicle to cross a road during optimal conditions, i.e. at the maximum permitted speed with no delays. TTI is sometimes referred to as the congestion index [7] as it is a useful metric

\footnotetext{
${ }^{1}$ http://sumo.dlr.de/index.html
} 
for measuring congestion levels in a given road network, it is calculated as described in Eq. 7.

$$
T T I=\frac{T T_{\text {average }}}{T T_{\text {average }}-\text { timeloss }}>=1
$$

where timeloss refers to the time lost (i.e., the additional travel time) due to driving below the ideal speed.

Before measuring the above performance metrics using CRITIC algorithm, a baseline was generated to measure these metrics in simulation scenarios where CRITIC mechanism is disabled. All simulations were run several times with a different random seed to get statistically meaningful results.

\section{B. Simulation scenarios}

1) Grid road network simulation: Grid networks can be found in many large cities worldwide, with the most notable example being New York City road network. In this network, all of the roads connect in a grid form, forming a pattern of rectangles and squares. This topology can be very effective at reducing congestion as less complex joins occur between the road intersections, meaning vehicles can travel across the junctions easier, forming less traffic build up. However, due to its Grid style such network can still be over-congested, as the number of vehicles exceeds the physical capacity of the network, traffic congestion begins to build. To mimic the topology of this network, we generate a 10x10 GRID network, consisting of multiple $3 \times 3$ lanes, with the outer most lane being a priority lane (i.e., a reserved lane for buses for example). This simulation was mainly built to assess CRITIC's ability to alleviate the congestion level in congested Grid networks, with the hypothesis that this algorithm could help improve traffic throughput for heavily congested intersections, by opening up new lanes.

In order to assess how well CRITIC performs under varying congestion levels, we have simulated three scenarios representing three different traffic loads, 500, 1000 and 2000 vehicles, each with additional bus traffic generated at 20 step intervals. The delays between buses provide time for CRITIC algorithm to optimize the road network usage, providing opportunities for vehicles to make use of these priority lanes. The bar chart plotted in Figure 4 shows the obtained ATT results from the three simulation scenarios described above. Once the statistics are produced from each scenario, the three files produced by SUMO are concatenated together, providing a larger more reliable dataset to analyze. As we can see, overall CRITIC achieved a notable decrease in ATT in all three simulation scenarios, reducing the ATT by more than 50 seconds in each setting.

The histogram depicted in Figure 5 presents the distribution of the achieved travel times in the GRID networks for both baseline and CRITIC. This figure reveals that CRITIC algorithm produces a large sample of shorter travel times in comparison

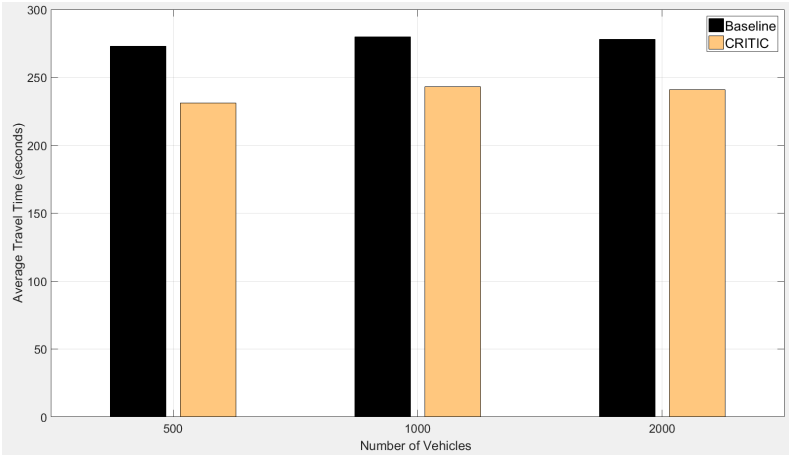

Figure 4: Average Travel Time in 10x10 Grid network: Baseline vs. CRITIC

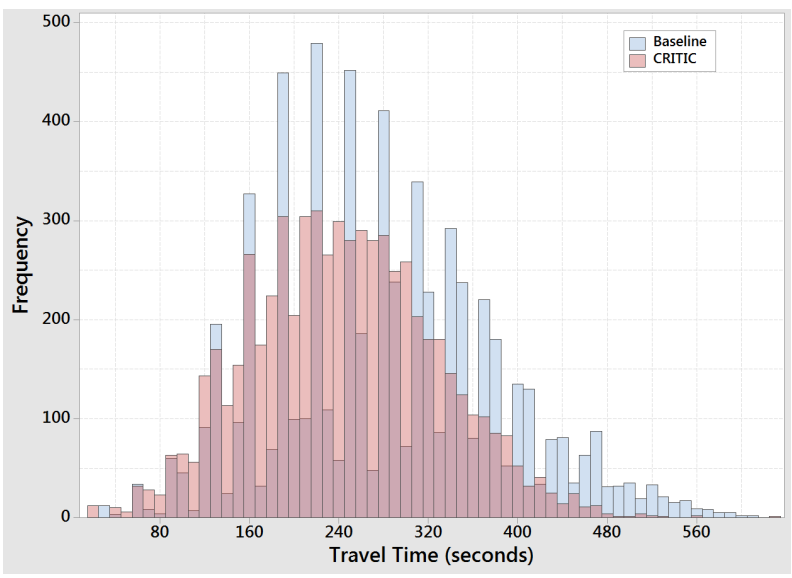

Figure 5: Trips duration distribution in 10x10 Grid network: Baseline vs. CRITIC

to the baseline simulation, with fewer extreme travel times. This is a direct consequence of the opportunistic usage of the bus lanes by the vehicles selected by CRITIC algorithm, thereby reducing their trip time as well as that of other vehicles using the regular lanes.

\begin{tabular}{|c|c|}
\hline Simulation & $\%$ improvement of priority lanes' ATT \\
\hline GRID-500 & -25.80 \\
\hline GRID-1000 & -28.85 \\
\hline GRID-2000 & -19.50 \\
\hline
\end{tabular}

Table I: The achieved improvement of ATT in priority lanes: 10x10 GRID network (-: means a reduction of the ATT)

Alongside producing positive results for the overall ATT, the GRID simulation also provided more insightful data in terms of the effects CRITIC would have on the priority lanes and their intended purpose. Table I shows the ATT improvement achieved in each simulation scenario, as we can see the largest improvement in travel time was $28.85 \%$, proving that CRITIC does not only improve the overall network performance, but also the performance of the priority lanes themselves. The data 
shown in this table represents the achieved physical ATT ${ }^{2}$ of the priority lanes (not the ATT of the vehicles that used these lanes). This statistic is more relevant to proving that CRITIC does not impact travel times in bus lanes because SUMO implements real world bus policies, meaning that buses do not always use bus lanes, they can also use non-priority lanes when required, in such cases as turning at a junction.

\begin{tabular}{|c|c|}
\hline Simulation & $\%$ improvement of TTI \\
\hline GRID-500 & -15.05 \\
\hline GRID-1000 & -13.62 \\
\hline GRID-2000 & -12.91 \\
\hline
\end{tabular}

Table II: The achieved improvement of TTI: 10x10 GRID network

Finally, TTI was also calculated for the GRID network, showing how CRITIC algorithm reduced the overall TTI for each simulation. Table II shows the achieved results with the greatest reduction being at a density of 500 vehicles, the reduction means that not only is travel time faster, but at the networks optimum free flow capacity, the journey time is actually closer to the optimum.

2) Abstract road network simulation: In this scenario, we simulate an abstract topology of a road network that mimics a more complex, real road network.

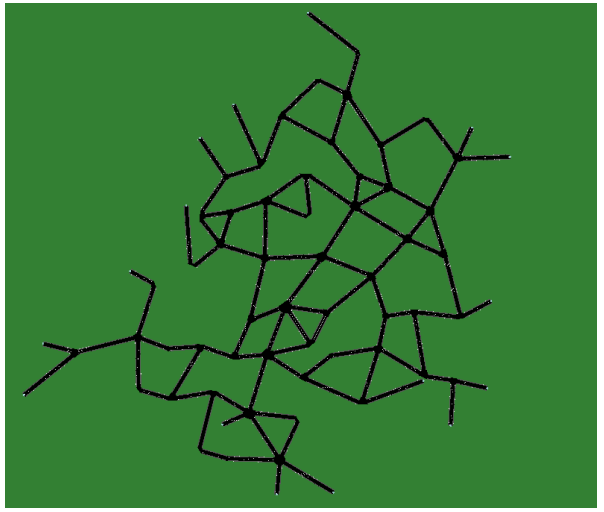

Figure 6: Abstract road network topology generated by SUMO

From Figure 6 we can clearly see how an abstract road network attempts to mimic the natural road networks available in many cities, with multiple different junctions and connecting lanes, with no uniformity between each edge. In order to maintain the same test environment as the Grid network simulation, the same vehicular densities were reproduced, ranging from 500 to 2000 vehicles.

The statistics collected from the abstract network simulation are plotted in Figures 7 and 8. The bar chart depicted in Figure 7 shows how the ATT of the baseline simulations

\footnotetext{
${ }^{2}$ The physical ATT refers to the average travel time of all vehicles that crossed a given priority lane while crossing this latter. In other words, it is a measure of how fast these vehicles crossed the priority lane.
}

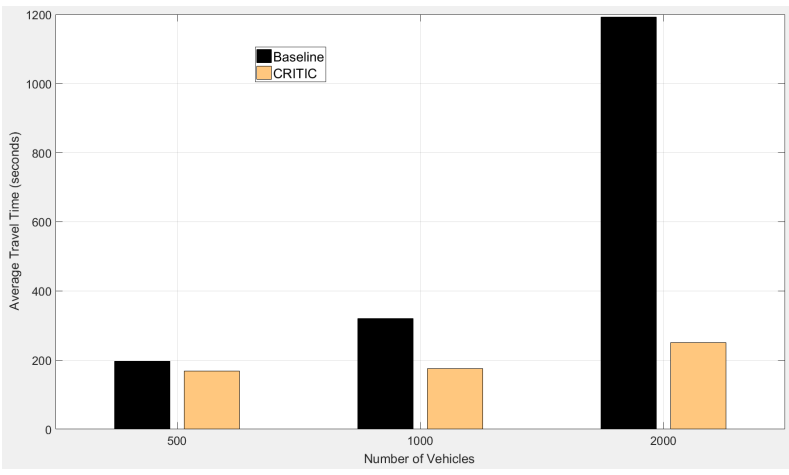

Figure 7: Average Travel Times in in an abstract road network topology: Baseline vs. CRITIC

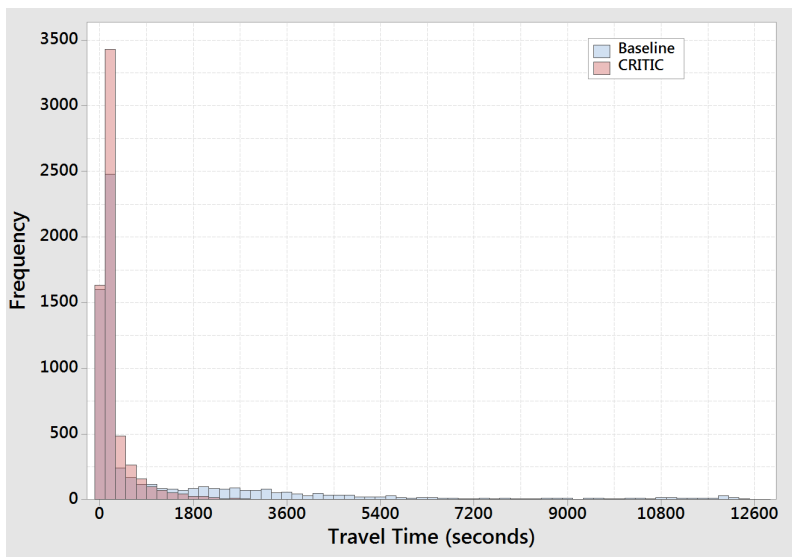

Figure 8: Trips duration distribution in an abstract road network topology: Baseline vs. CRITIC

began to increase drastically as the number of vehicles in the simulation doubled, but CRITIC was only slightly affected by the same change. The histogram plotted in Figure 8 shows the results obtained from the most performant simulation, which was a density of 2000 vehicles. This histogram compares all the achieved travel times of each vehicle in the network for baseline and CRITIC simulations. These results reveal that the greater majority of vehicles achieved much shorter travel times in comparison with the baseline results, while also producing less extreme results, which can be caused by extreme congestion or road incidents. The above results highlight also that since the buses can utilize all lanes in a road network, CRITIC algorithm reduces the overall congestion on the network and, therefore, improves the travel time of priority vehicles as well.

\begin{tabular}{|c|c|}
\hline Simulation & \% ATT of priority vehicles \\
\hline ABSTRACT-500 & -16.27 \\
\hline ABSTRACT-1000 & -26.98 \\
\hline ABSTRACT-2000 & -59.71 \\
\hline
\end{tabular}

Table III: The achieved improvement of ATT for priority vehicles: Abstract network 
Table III shows the percentage improvement of the ATT for all priority vehicles in the abstract network. We can observe that as the network becomes more congested, CRITIC algorithm achieves significant reduction in travel time, with an almost $60 \%$ reduction for the abstract-2000 simulation scenario. This is a very promising result as it highlights that once a road network becomes extremely congested, all vehicles benefit from the reduced congestion, due to the usage of CRITIC, of non-priority lanes.

\begin{tabular}{|c|c|}
\hline Simulation & \% improvement of TTI \\
\hline ABSTRACT-500 & -19.95 \\
\hline ABSTRACT-1000 & -51.42 \\
\hline ABSTRACT-2000 & -75.99 \\
\hline
\end{tabular}

Table IV: The achieved improvement of TTI: Abstract network

The Abstract network also provides extremely positive TTI results for CRITIC algorithm, as the table above shows in the most congested network of 2000 vehicles, CRITIC algorithm ensures a $75 \%$ reduction in TTI. This clearly highlights that CRITIC has the ability to reduce road traffic congestion on highly congested road networks, not only reducing overall travel times but also increasing the overall quality of service of the road network.

\section{CONCLUSION}

This paper has addressed one of the most pressing issues of the 21 st century, as cities grow and the human population increases to unforeseen levels, new challenges arise, which must be solved in order to maintain a productive and healthy society. Road traffic congestion is one of these issues, without an efficient traffic management system and modern road infrastructure, the economy and health of many cities will deteriorate, as business fails to grow and the environment begins to suffer. In contrast to numerous existing solutions, our original and cognitive radio inspired solution, dubbed CRITIC, attempts to provide evidence that not all changes need to be in the physical road network, but changes to laws and road policies can also bring profound effects to the efficiency of current road traffic networks. CRITIC's efficiency has been proven through extensive simulation, showing between $15 \%$ to $75 \%$ reduction in the overall travel time and a substantial increase of the number of shorter trips. We strongly believe that CRITIC could be the first step towards developing more sophisticated solutions that can revolutionize the upcoming era of driverless cars and smart road infrastructure.

\section{REFERENCES}

[1] S. Djahel, R. Doolan, G. M. Muntean and J. Murphy, "A Communications-Oriented Perspective on Traffic Management Systems for Smart Cities: Challenges and Innovative Approaches," in IEEE Communications Surveys \& Tutorials, vol. 17, no. 1, pp. 125-151, Firstquarter 2015.
[2] Litman, T. "Comprehensive Evaluation Of Traffic Congestion Costs and Congestion Reduction Strategies". Victoria Transport Policy Institute, April 2017. http://www.vtpi.org/cong_relief.pdf

[3] D. Schrank, B. Eisele, T. Lomax and J. Bak, "2015 URBAN MOBILITY SCORECARD," Published jointly by The Texas A\&M Transportation Institute and INRIX, August 2015.

[4] D.A. Hennessy and D.L. Wiesenthal, "Traffic Congestion, Driver Stress, and Driver Aggression," Aggressive Behavior Journal, Vol. 25, No. 6, pp. 409-423, 1999.

[5] R.W. Novaco and O. Gonzalez, "Commuting and wellbeing," in Y. Amichai-Hamburger (Ed). Technology and well-being (pp. 174-205). Cambridge: Cambridge University Press, Jan. 2009.

[6] R.W. Novaco, D. Stokols, J. Campbell and J. Stokols, "Transportation, Stress, and Community Psychology". American Journal of Community Psychology, Vol. 7, No. 4, pp. 361-380, August 1979.

[7] S. Wang, S. Djahel, Z. Zhang and J. McManis, "Next Road Rerouting: A Multiagent System for Mitigating Unexpected Urban Traffic Congestion," in IEEE Transactions on Intelligent Transportation Systems, vol. 17, no. 10, pp. 2888-2899, Oct. 2016.

[8] S. Faye, C. Chaudet and I. Demeure, "A Distributed Algorithm for Adaptive Traffic Lights Control in Wireless Sensor Networks," in Proceedings of the first workshop on Urban networking (UrbaNe '12), Dec. 10, 2012, Nice, France.

[9] V.T. Nguyen, F. Villain and Y. Le Guillou, "Cognitive radio RF: overview and challenges," VLSI Design, vol. 2012, Article ID 716476, 13 pages, 2012. doi:10.1155/2012/716476

[10] N. OHara, M. Slot, J. Monteil, V. Cahill and M. Bouroche, "Towards Evaluating the Benefits of Intervehicle Coordination," 2015 IEEE 18th International Conference on Intelligent Transportation Systems, Las Palmas, 2015, pp. 2444-2450.

[11] U. Khan, P. Basaras, L. Schmidt-Thieme, A. Nanopoulos and D. Katsaros, "Analyzing cooperative lane change models for connected vehicles," 2014 International Conference on Connected Vehicles and Expo (ICCVE), Vienna, 2014, pp. 565-570.

[12] D. Schrank, B. Eisele and T. Lomax, "TTI's 2012 urban mobility report", Texas Transp. Inst., Texas A \& M Univ., College Station, TX, USA, 2012.

[13] S. Haykin, "Cognitive radio: brain-empowered wireless communications," in IEEE Journal on Selected Areas in Communications, vol. 23, no. 2, pp. 201-220, Feb. 2005. doi: $10.1109 /$ JSAC. 2004.839380 